\title{
Clinical efficacy and safety of mucosal incision-assisted biopsy for the diagnosis of upper gastrointestinal subepithelial tumors: A systematic review and meta-analysis
}

\author{
Amaninder Dhaliwala, Sindhura Kollib, Banreet Singh Dhindsac, Kalpit Devani ${ }^{d}$, Daryl Ramaie, \\ Harlan Sayles, , Rajani Rangray ${ }^{g}$, Ishfaq Bhat ${ }^{a}$, Shailender Singh ${ }^{a}$, Douglas G. Adler
}

University of Nebraska Medical Center; NYU Langone Medical Center, NY; University of Nevada Las Vegas School of Medicine, Las Vegas; East Tennessee University, Johnson City, TN; The Brooklyn Hospital Center, NY; CHI-Creighton University Medical Center, Omaha, NE; University of Utah School of Medicine, Huntsman Cancer Center, Salt Lake City, Utah, USA

Abstract

\begin{abstract}
Background Endoscopic ultrasound-guided fine-needle aspiration and biopsy (EUS-FNA/FNB) has been traditionally used for making a tissue diagnosis. Several newer techniques are emerging as a viable alternative to EUS-FNA/FNB, including mucosal incision-assisted biopsy (MIAB), with a view to increasing the diagnostic yield for upper gastrointestinal (GI) subepithelial tumors (SETs). We conducted a systematic review and meta-analysis to describe the overall diagnostic yield of MIAB for upper GI SETs.

Methods Multiple electronic databases (MEDLINE, EMBASE and Google Scholar) and conference abstracts were comprehensively searched. The primary outcome of our meta-analysis was the overall diagnostic yield of the MIAB. The secondary outcome was to study complications in terms of perforation and clinically significant bleeding. The meta-analysis was performed using a DerSimonian and Laird random-effect model.

Results Seven studies were included in the final meta-analysis, reporting a total of 159 patients (male 86, female 73) with a mean age of 58 years. The overall pooled diagnostic yield of MIAB was $89 \%$ (95\% confidence interval [CI] 82.65-93.51, $I^{2}=0.00$ ). Histologically, GI stromal tumor was the reported diagnosis in $38.62 \%$ (95\% CI $22.29-56.24, I^{2}=77.51 \%$ ) of tumors, followed by leiomyoma $25 \%$ (95\%CI $\left.18.02-32.62, I^{2}=4.42 \%\right)$. The overall rate of clinically significant bleeding following the procedure was $5.03 \%\left(95 \% \mathrm{CI} 0.36-12.86, I^{2}=57.43 \%\right)$ and no perforations were reported.

Conclusions MIAB is a safe and effective technique for the diagnosis of upper GI SETs and can be considered as a viable alternative to EUS-FNA/FNB. MIAB can be performed during routine endoscopy and no advanced equipment is required.
\end{abstract}

Keywords Mucosal, incision, biopsy, subepithelial, gastrointestinal

Ann Gastroenterol 2020; 33 (1): 1-7 a Division of Gastroenterology and Hepatology, University of Nebraska Medical Center (Amaninder Dhaliwal, Ishfaq Bhat, Shailender Singh); ${ }^{b}$ Department of Medicine, NYU Langone Medical Center, NY (Sindhura Kolli); 'Department of Internal Medicine, University of Nevada Las Vegas School of Medicine, Las Vegas (Banreet Singh Dhindsa); ${ }^{\mathrm{d}}$ Department of Gastroenterology, East Tennessee University, Johnson City, TN (Kalpit

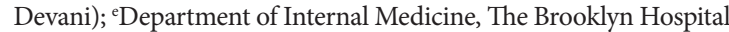
Center, Brooklyn, NY (Daryl Ramai); f Department of Biostatistics, University of Nebraska Medical Center, Omaha (Harlan Sayles); ${ }^{\mathrm{g}}$ Division of Gastroenterology and Hepatology, CHI-Creighton University Medical Center, Omaha, NE (Rajani Rangray); ${ }^{\mathrm{h}}$ Department of Gastroenterology and Hepatology, University of Utah School of Medicine, Huntsman Cancer Center, Salt Lake City, Utah (Douglas G. Adler), USA

\section{Conflict of Interest: None}

Correspondence to: Douglas G Adler, MD, FACG, AGAF, FASGE, Professor of Medicine, Director of Therapeutic Endoscopy, Director, GI fellowship program, Gastroenterology and Hepatology University of Utah School of Medicine, Huntsman Cancer Center, 30 N 1900 E, Room 4R118, Salt Lake City, Utah 84132, USA, e-mail: douglas.adler@hsc.utah.edu

Received 18 Novmeber 2019; accepted 22 January 2020; published online 14 February 2020

DOI: https://doi.org/10.20524/aog.2020.0460

๑ 2020 Hellenic Society of Gastroenterology

\section{Introduction}

Gastrointestinal (GI) subepithelial tumors (SETs) represent only a small proportion $(<1 \%)$ of all GI tumors and have a wide spectrum of presentations, pathologies, and prognosis [1,2].GI SETs include both non-neoplastic and neoplastic lesions. To delineate between high-risk lesions such as GI stromal tumors (GISTs) (which have a 30\% potential for malignancy), and low-risk lesions such as lipomas, tests with high diagnostic yield and accuracy are essential $[1,3,4]$.

Standard endoscopy helps in the observational analysis of the size, margins, color and possible level of involvement within the wall of upper GI SETs [5]. However, upper GI SETs are by definition below the mucosa; hence, for the purposes of preoperative diagnosis, invasive methods that include tissue sampling for cytology, histology and immunohistochemical analysis are often required $[5,6]$.

The current standard for obtaining tissue samples to differentiate between types of upper GI SETs is an endoscopic ultrasound-guided fine-needle aspiration (EUS-FNA). It 
has achieved gold standard status because of its diagnostic accuracy of $100 \%$ in lesions bigger than $40 \mathrm{~mm}$. However, the accuracy of EUS-based approaches falls as lesions diminish in size, with accuracy falling to as low as $50 \%$ in lesions smaller than $20 \mathrm{~mm}$ [7]. This variability in accuracy, along with other drawbacks, such as the cost of EUS-FNA, the average time it entails, and the required level of expertise required from an advanced endoscopist, has left the window open for improvements and alternative tissue sampling modalities [8].

As the evolution of diagnostic methods for upper GI SETs continues, alternatives such as mucosal incision assisted biopsy (MIAB) have been developed, with a reported diagnostic yield of $89 \%$ [8]. This technique was developed by Yokohata et al in 2007. In this method, saline is injected through the mucosa to create a submucosal fluid cushion in a manner similar to that used during salineassisted polypectomy or endoscopic submucosal dissection. A needle knife or related device is then used to incise the mucosa and the submucosa until the GI SET is reached. Then, using cold forceps an average of 3-6 tissue samples are obtained $[8,9]$.

The duration of the procedure is similar to or less than the duration of an EUS-FNA. It is less expensive than an EUS-FNA, able to maneuver into locations that can be challenging with an EUS-FNA, and does not require advanced endoscopic training [8]. No instances of perforation have been reported thus far [5,8,10-15]. Many are unaware of this technique or have not been exposed to it in a meaningful way. The aim of this systematic review and meta-analysis will be to analyze the overall diagnostic yield and complications of MIAB compared to the current standard of EUS/FNA.

\section{Materials and methods}

The objectives, primary and secondary outcomes, search strategy, inclusion and exclusion criteria, and methods for study selection, data extraction, and data synthesis of this meta-analysis were defined in a protocol in advance as per guidelines [16,17].

\section{Information sources and search strategy}

A literature search was performed within the databases of PubMed, Cochrane Library and Web of Science. Various amalgamations of the following keywords were utilized: "mucosal incision assisted biopsy," "intraepithelial gastric lesions," "mucosal incision biopsy," "EUS-FNA," "GISTs," "stromal tumors," "upper gastrointestinal subepithelial tumors," "submucosal lesion," "EUS," and "EGD." Relevant studies chosen were published between August 2007 and August 2017.

\section{Inclusion and exclusion criteria}

We followed the Preferred Reporting items for Systematic Reviews and Meta-Analyses (PRISMA) guidelines, using a predefined protocol to identify studies that reported the diagnostic yield of MIAB for upper GI SETs (Fig. 1) [18]. Study designs included comprised prospective and retrospective studies, as well as abstracts presented at national meetings. Studies with incomplete data and those not meeting the inclusion criteria were excluded.

\section{Data extraction and quality assessment}

Data were extracted and verified by independent reviewers. The initial screening stage consisted of searching for studies in which the title and abstract delineated evaluation of the diagnostic yield of MIAB of upper GI SETs in human subjects. In the subsequent stage, a complete examination of the article was undertaken to ensure its relevance to our points of interest. Data collected included the first author, year of publication, years the study spanned, type of study, total number of patients, size and type of upper GI SETs, and diagnostic yield of MIAB. We assessed the quality of included studies using a scale modified from the Newcastle--Ottawascalefor cohort studies [18].

\section{Outcomes assessed}

The primary outcome was the overall diagnostic yield of MIAB in the diagnosis of upper GI SETs. It was defined as the proportion of patients for whom the pathologist could provide a definitive diagnosis based on the specimen provided [15].

The secondary outcome was to analyze adverse events, including but not limited to perforation and clinically significant bleeding. Clinically significant bleeding was defined as any bleeding requiring blood transfusion or endoscopic intervention $[5,15]$.

\section{Statistical analysis}

We used meta-analysis techniques to calculate the pooled rates in each case, following the methods suggested by DerSimonian and Laird and using the random-effects model [18]. All pooled rates were calculated with a 95\% confidence interval (CI). A forest plot was constructed for the pooled estimates of both the primary outcome, diagnostic yield of MIAB, and the secondary outcomes. The variation of results across studies due to heterogeneity rather than chance was expressed by $I^{2}$. $I^{2}$ values of $<30 \%, 30-60 \%, 61-75 \%$ and $>75 \%$ were suggestive of low, moderate, substantial and considerable heterogeneity, respectively. 


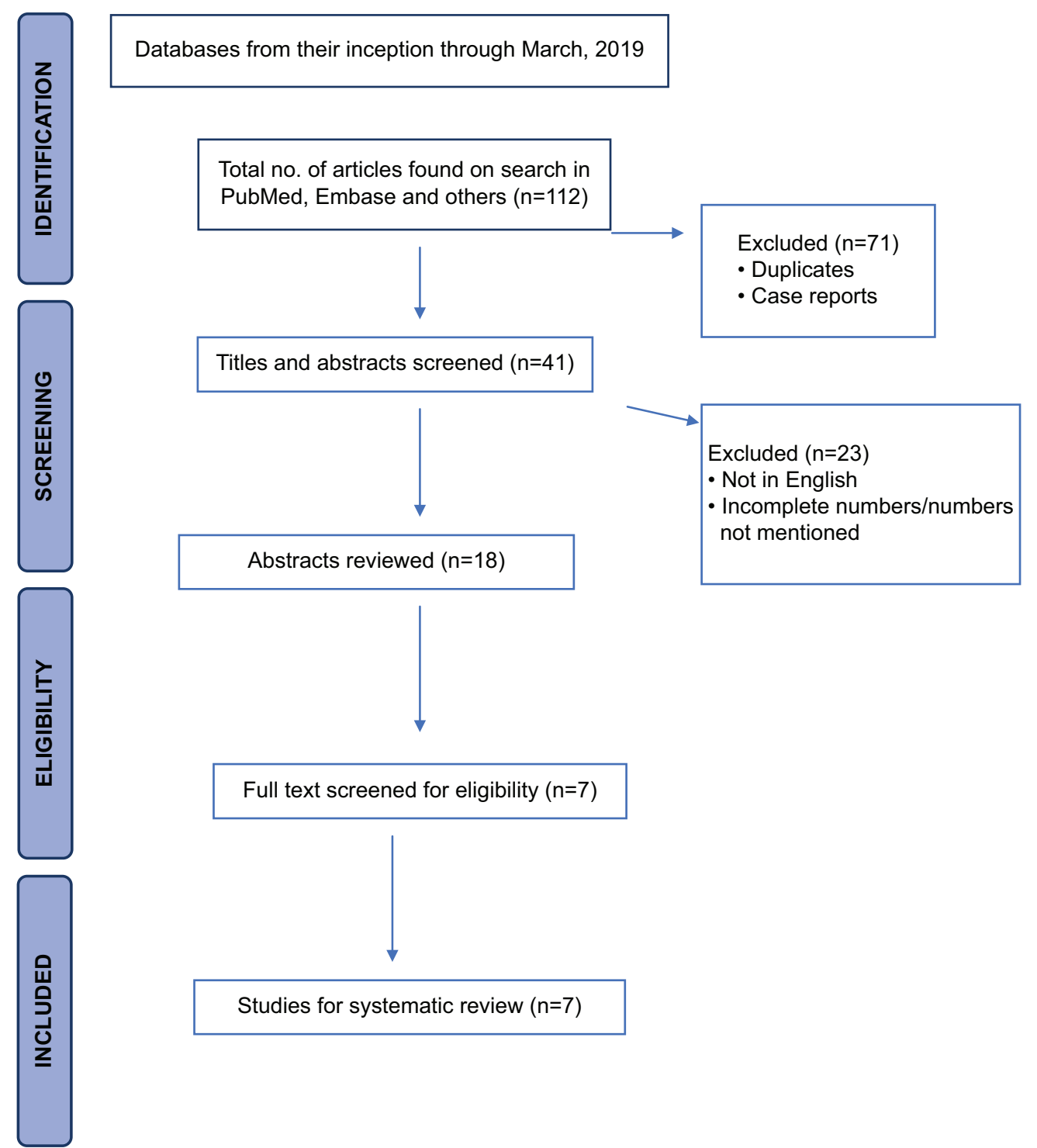

Figure 1 Study selection process in accordance with preferred reporting items for systematic reviews and meta-analysis statement (PRISMA)

\section{Results}

\section{Literature search}

Using our search criteria, a total of 41 citations were identified. After duplicate studies were removed, 23 citations were reviewed. On final application of the inclusion and exclusion criteria, 7 studies that discussed the use of MIAB in the diagnosis of upper GI SETs were included. The meta-analysis thus included 7 independent cohort studies totaling 159 patients (Fig. 1). All of them were single-center based. Three studies contained 27 or more patients, while 4 had between 12 and 15. Six studies were retrospective and one was prospective. Five studies were full publications and in original manuscript form. The remaining 2 studies were in abstract form and are still pending full publication. Of the 159 patients, $86(54.1 \%)$ were male and $73(45.9 \%)$ were female, with a mean age of 58 years. Study details are shown in Table 1.

\section{Overall diagnostic yield}

In our study population, the overall pooled diagnostic yield of MIAB for upper GI SETs was $89 \%$ (95\%CI 82.65-93.51, $I^{2}=0.00 \%$ ), as outlined in the forest plot (Fig. 2). GISTs were diagnosed in $38.62 \%$ of these $(95 \% \mathrm{CI}$ 22.29-56.24, $I^{2}=77.51 \%$ ), followed by leiomyomas $25 \%$ (95\%CI 18.02-32.62, $\left.I^{2}=4.42 \%\right)$. The mean tumor size was $21.0 \mathrm{~mm}$ (95\%CI 18.1-24.0, $\left.I^{2}=89.73 \%\right)$. A majority of the upper GI SETs were located in the stomach $(94.76 \%, 95 \%$ CI $78.46-100$, $\left.I^{2}=84.49 \%\right)$. 


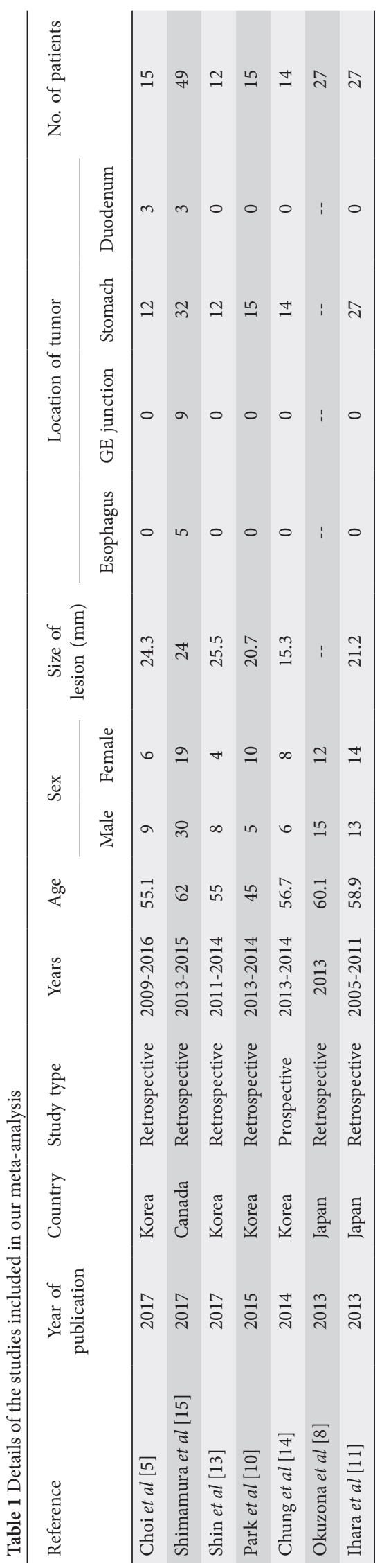

\section{Adverse events}

No perforations were not reported in any of the 7 studies. Overall, the rate of clinically significant bleeding following MIAB was $5.03 \%$ (95\%CI $\left.0.36-12.86, I^{2}=57.43 \%\right)$, as outlined in the forest plot (Fig. 3). A majority of bleeding events were immediate and controlled with endoscopic hemostatic measures.

\section{Validation of meta-analysis results}

\section{Heterogeneity}

Based on Q statistics and $I^{2}$ analysis for heterogeneity, no heterogeneity was observed in the overall pooled diagnostic yield of MIAB for upper GI SETs. Within the category of upper GI SETs, considerable heterogeneity was noted in the diagnostic yield of GISTs $\left(I^{2}=77.51 \%\right)$, the mean tumor size $\left(I^{2}=89.73 \%\right)$, and the tumor location $\left(I^{2}=84.49 \%\right)$. Moderate heterogeneity $\left(I^{2}=57.43 \%\right)$ was noted in the rates of clinically significant bleeding.

\section{Publication bias and quality of the studies}

The power to detect publication bias was low, given the small number of studies for comparison. The quality of the studies was assessed by the Newcastle--Ottawa scale (Table 2). Overall, 4 studies were considered to be of high quality and 3 studies were of low quality. None of the studies were of medium quality.

\section{Discussion}

EUS-FNA is an established diagnostic modality for the investigation of upper GI SETs. It has attained this status because of its accuracy when dealing with large lesions and its minimal complication rate when performed by an experienced advanced endoscopist. Disadvantages of EUSFNA include the diminishing diagnostic yield with smaller or difficult-to-reach GI SETs, the cost of the procedure, the need for it to be performed by a skilled advanced endoscopist, and the long procedure time. This systematic review of studies involving MIAB demonstrate this method's high diagnostic yield for upper GI SETs and its minimal complications, making it a viable alternative to EUS-FNA in the approach to upper GI SETs in centers that do not have an advanced endoscopist.

Our results showed a pooled diagnostic yield of $89 \%$ (95\%CI 82.65-93.51, $I^{2}=0.00 \%$ ) for upper GI SETs using the MIAB technique. In a study by Okuzono et al, the procedure took approximately $20 \mathrm{~min}$, during which on average 6 biopsies were obtained [8]. Ihara et al noted that MIAB only required an electrosurgical generator and electrosurgical knives, and the skills of a general 


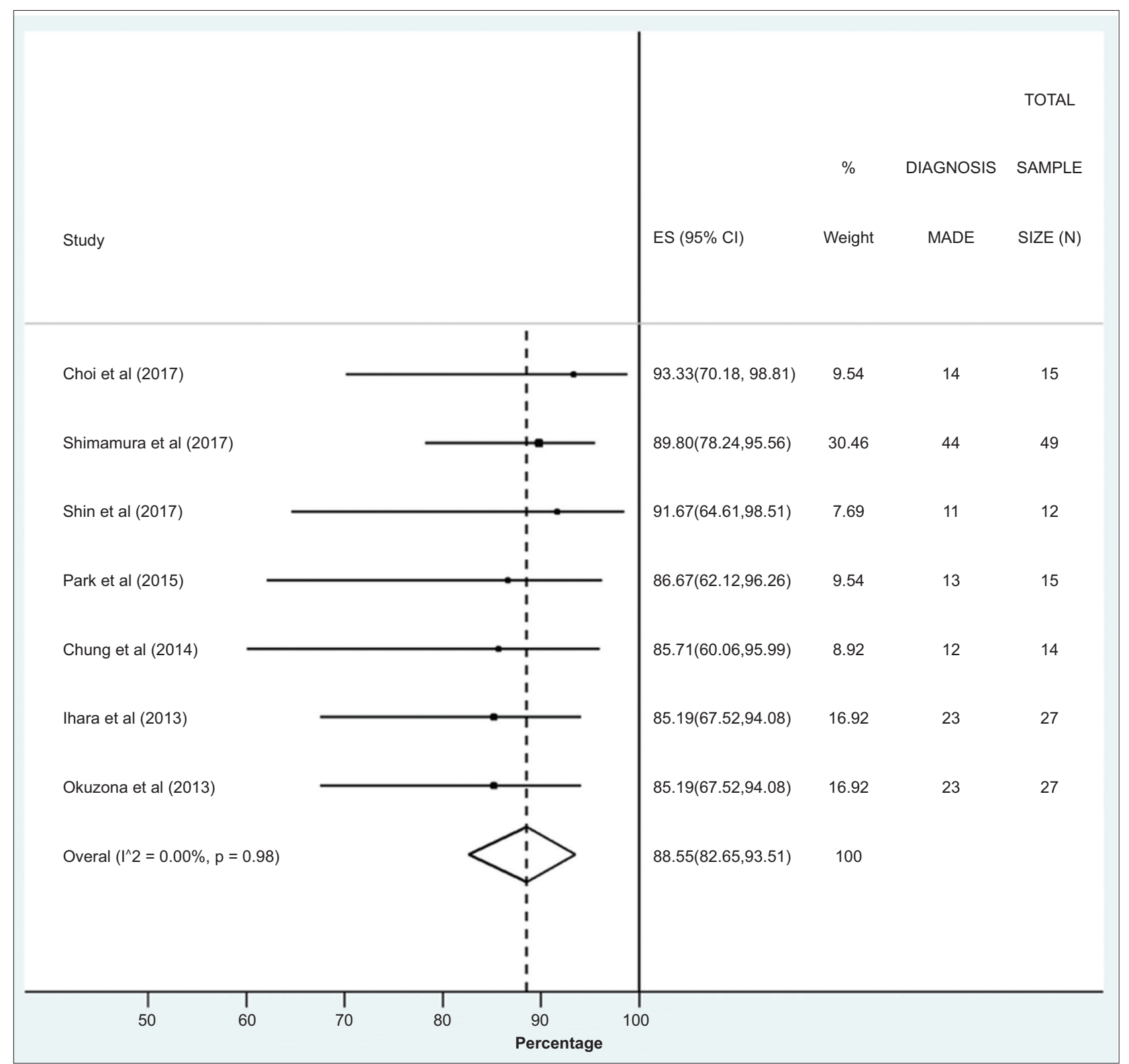

Figure 2 Overall pooled diagnostic yield of mucosal incision assisted biopsy

ES, effect size; CI, confidence interval; $N$, number within sample size, p, probability value; $I^{2}$, heterogeneity

Table 2 Newcastle-Ottawa scale for study quality assessment

\begin{tabular}{|c|c|c|c|c|c|c|}
\hline \multirow[t]{2}{*}{ Author } & \multirow[t]{2}{*}{ Study type } & \multirow[t]{2}{*}{ Cohort/Case-control } & \multirow[t]{2}{*}{ Year } & \multicolumn{2}{|c|}{ Newcastle-Ottawa scale } & \multirow[t]{2}{*}{ Outcomes } \\
\hline & & & & Selection & Comparability & \\
\hline Shin et al [13] & Retrospective & Cohort & 2017 & $* * *$ & * & * \\
\hline Choi et al [5] & Retrospective & Cohort & 2017 & $* * *$ & * & * \\
\hline Shimamura et al [15] & Retrospective & Cohort & 2017 & $* * *$ & * & $* *$ \\
\hline Park et al [10] & Retrospective & Cohort & 2015 & $* * * *$ & * & ** \\
\hline Chung et al [14] & Prospective & Cohort & 2014 & $* * *$ & * & * \\
\hline Ihara et al [11] & Retrospective & Cohort & 2013 & $* * *$ & * & ** \\
\hline Okuzona et al [8] & Retrospective & Cohort & 2013 & $* * * *$ & $* *$ & ** \\
\hline
\end{tabular}




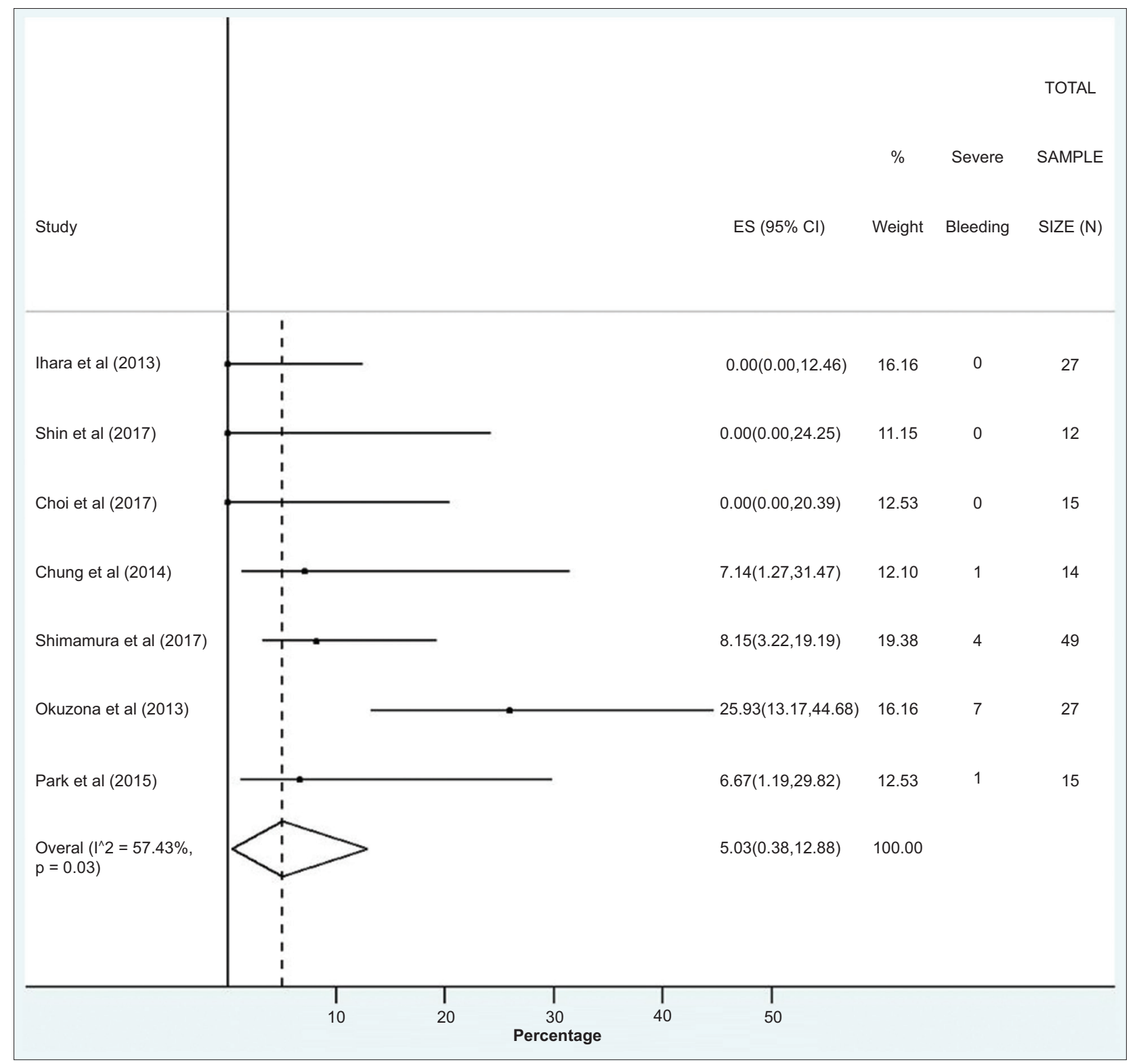

Figure 3 Rate of clinically significant bleeding following Mucosal incision assisted biopsy

$E S$, effect size; CI, confidence interval; $N$, number within sample size, p, probability value; $I^{2}$, heterogeneity

gastroenterologist, whereas an EUS-FNA requires a linear echoendoscope, the skills of an advanced endoscopist, and, in some institutions, a cytotechnologist or cytologist for rapid onsite evaluation (ROSE) of the specimens, which can improve the diagnostic yield of an EUS-FNA sample by $24 \%$ on average $[11,12]$.

In our meta-analysis, MIAB had a bleeding rate of 5.03\% (95\%CI $0.36-12.86, I^{2}=57.43 \%$ ). One study reported a case of intraoperative bleeding that prevented the endoscopist from obtaining a sufficient number of tissue samples, while another reported delayed bleeding due to a deep biopsy $[8,10]$. No perforations were reported in any of the 7 studies. Multiple studies noted that MIAB was a safe technique, with minimal bleeding and potentially a lower risk of complications when used on intraluminal versus extraluminal growths $[8,11,13]$.

Limitations of the included studies were that they were all single-center studies with small sample sizes (under 49 patients) and moderate-to-considerable heterogeneity.

Overall, MIAB is an effective and safe procedure for diagnosing GI SETs, with an overall diagnostic yield of $89 \%$ and a low rate of adverse events. This technique does not require advanced endoscopy training and can be performed by general gastroenterologists, potentially using fewer resources. Multi-center randomized control trials are needed to further validate these findings. 


\section{Summary Box}

\section{What is already known:}

- Endoscopic ultrasound-guided fine-needle aspiration and biopsy (EUS-FNA/EUS-FNB) is currently the gold standard for tissue diagnosis of upper gastrointestinal (GI) subepithelial tumors (SETs)

- EUS-FNA has a diagnostic accuracy of $100 \%$ in lesions larger than $40 \mathrm{~mm}$; in lesions, between $20 \mathrm{~mm}$ and $40 \mathrm{~mm}$ its accuracy diminishes to $86-91 \%$; and in lesions smaller than $20 \mathrm{~mm}$ it becomes $<50 \%$

- This variability in accuracy and other drawbacks, such as the cost of EUS-FNA, the average time it entails, and the level of expertise required from an advanced endoscopist, have left the window open for improvements and alternative tissue sampling modalities

\section{What the new findings are:}

- Mucosal incision-assisted biopsy is emerging as an alternative technique for the tissue diagnosis of upper GI SETs, with an overall diagnostic yield of $89 \%$

- The duration of the procedure is similar to or less than the duration of an EUS-FNA

- It is less expensive than an EUS-FNA, able to maneuver into locations that can be challenging with an EUS-FNA, and does not require advanced endoscopic training

- It is an overall safer technique with minimal bleeding rates of $5 \%$ managed endoscopically, with no perforations have been noted

\section{References}

1. Hwang JH, Rulyak SD, Kimmey MB; American Gastroenterological Association Institute. American Gastroenterological Association Institute technical review on the management of gastric subepithelial masses. Gastroenterology 2006;130:2217-2228.

2. Zhao X, Yue C. Gastrointestinal stromal tumor. J Gastrointest Oncol 2012;3:189-208.

3. Franco MC, Schulz RT, Maluf-Filho F. Opinion: How to manage subepithelial lesions of the upper gastrointestinal tract? World J Gastrointest Endosc 2015;7:1262

4. Søreide K, Sandvik OM, Søreide JA, Giljaca V, Jureckova A, Bulusu VR. Global epidemiology of gastrointestinal stromal tumours (GIST): A systematic review of population-based cohort studies. Cancer Epidemiol 2016;40:39-46.

5. Choi CW, Kang DH, Kim HW, et al. Direct endoscopic biopsy for subepithelial tumor larger than $20 \mathrm{~mm}$ after removal of overlying mucosa. Scand J Gastroenterol 2017;52:779-783.

6. Yamabe A, Irisawa A, Bhutani MS, et al. Usefulness of endoscopic ultrasound-guided fine-needle aspiration with a forward-viewing and curved linear-array echoendoscope for small gastrointestinal subepithelial lesions. Endosc Int Open 2015;3:E161-E164.

7. Attila T, Aydin O. Lesion size determines diagnostic yield of EUS-FNA with onsite cytopathologic evaluation for upper gastrointestinal subepithelial lesions. Turk $J$ Gastroenterol 2018;29:436-441.

8. Okuzono T, Mishima T, Badran A, et al. Endoscopic biopsy by mucosal incision for upper gastrointestinal submucosal tumors. Video Journal and Encyclopedia of GI Endoscopy 2014;1:644-646.

9. Yokohata N, Tamegai Y, Tokuhara M, et al. Three cases of gastric submucosal tumor diagnosed preoperatively: direct mucosal incision biopsy for gastrointestinal submucosal tumors; Progress of Digestive Endoscopy 2007;70:82-83.

10. Park SE AJ, Jung HY, Jung KW, et al. Diagnostic yield of mucosal incision with deep biopsy for small gastric subepithelial tumor: a comparison with endoscopic ultrasound-guided fine needle aspiration. Presented at: $23^{\text {rd }}$ United European Gastroenterology Week; Oct 2015; Barcelona, Spain.

11. Ihara E, Matsuzaka H, Honda K, et al. Mucosal-incision assisted biopsy for suspected gastric gastrointestinal stromal tumors. World J Gastrointest Endosc 2013;5:191-196.

12. Alsohaibani F, Girgis S, Sandha GS. Does onsite cytotechnology evaluation improve the accuracy of endoscopic ultrasound-guided fine-needle aspiration biopsy? Can J Gastroenterol 2009;23:26-30.

13. Shin SY, Lee SJ, Jun JH, et al. Mucosal incision and forceps biopsy for reliable tissue sampling of gastric subepithelial tumors. Clin Endosc 2017;50:64-68.

14. Chung J SY. Mucosal-incision assisted biopsy for suspicious gastrointestinal stromal tumors. Presented at: $22^{\text {rd }}$ United European Gastroenterology Week; Oct 2014; Vienna, Austria.

15. Shimamura Y, Hwang J, Cirocco M, May GR, Mosko J, Teshima CW. Efficacy of single-incision needle-knife biopsy for sampling subepithelial lesions. Endosc Int Open 2017;5:E5-E10.

16. Stroup DF, Berlin JA, Morton SC, et al. Meta-analysis of observational studies in epidemiology: a proposal for reporting. Meta-analysis Of Observational Studies in Epidemiology (MOOSE) group. JAMA 2000;283:2008-2012.

17. Moher D, Liberati A, Tetzlaff J, Altman DG; PRISMA Group. Preferred reporting items for systematic reviews and metaanalyses: the PRISMA statement. Ann Intern Med 2009;151:264269, W64.

18. DerSimonian R, Laird N. Meta-analysis in clinical trials. Control Clin Trials 1986;7:177-188. 